(1)

George Fox

UNIVERSITY
Digital Commons @ George Fox University

Faculty Publications - School of Physical

Therapy

2018

Comparison of Jump and Hop Test Measures Between NAIA and

Division III Male Collegiate Basketball Players

Jason Brumitt

Amy Engilis

Alma Mattocks

Natalie Ellis

Jordon Reyes

Follow this and additional works at: https://digitalcommons.georgefox.edu/pt_fac

Part of the Physical Therapy Commons 


\title{
Comparison of Jump and Hop Test Measures Between NAIA and Division III Male Collegiate Basketball Players
}

\author{
Jason Brumitt, PT, PhD, ATC, CSCS; Amy Engilis, MA, ATC; Alma Mattocks, MS, ATC; Natalie Ellis, DPT; \\ Jordon Reyes, DPT
}

\begin{abstract}
Purpose: To report normative data for two functional performance tests (FPTs) (the standing long jump [SLJ] and the single-leg hop [SLH]) in a population of male collegiate basketball players and to identify differences in measures between athletes based on level of competition, starter status, and position.
\end{abstract}

Methods: Eighty-six male collegiate basketball players from six teams were recruited for this study. Each athlete performed three SLJs and three SLHs (bilaterally).

Results: Mean ( \pm SD) FPT measures (normalized to height) for all basketball players were: $\mathrm{SLJ}=1.0 \pm 0.1$, right $\mathrm{SLH}=0.84$ \pm 0.1 , and left SLH $=0.85 \pm 0.1$. Significant differences in FPT measures were observed both within and between groups based on: level of competition, by player position, and by starter status.

Conclusions: The data presented in this study can be used by coaches and athletic trainers to assess aspects of athletic readiness in male collegiate basketball players.

From the School of Physical Therapy, George Fox University, Newberg, Oregon (JB, NE, JR); Warner Pacific College, Portland, Oregon (AE); and Athletic Training Education Program, Spalding University, Louisville, Kentucky (AM).

Submitted: July 3, 2017; Accepted: January 3, 2018

The authors have no financial or proprietary interest in the materials presented herein.

Correspondence: Jason Brumitt, PT, PhD, ATC, CSCS, School of Physical Therapy, George Fox University, 414 N. Meridian Street, \#V123, Newberg, OR 97132. E-mail: jbrumitt@georgefox.edu

doi:10.3928/19425864-20180306-01 thletic trainers, strength coaches, and other sports
medicine professionals routinely evaluate perfor-
mance measures in athletes prior to the start of the sport season. ${ }^{1-10}$ These measures may be collected using "high-tech" tests (eg, maximal oxygen uptake or isokinetic testing) or "low-tech" tests (eg, functional performance tests [FPTs]). Advantages associated with the use of FPTs are that they are generally quick to perform, are inexpensive, and require minimal use of equipment. Functional performance testing has been used to collect measures associated with athletic readiness (eg, strength and agility), ${ }^{1-10}$ illustrate relationships between FPT measures and sportspecific tasks, ${ }^{10-13}$ identify athletes at risk for injury, ${ }^{14-20}$ and guide discharge from rehabilitation after an injury. ${ }^{21}$

However, only a few studies have reported preseason FPT measures and their relationships with performance variables or player position in male basketball players. McGill et al. ${ }^{13}$ tested torso endurance, hip range of motion, strength, speed, agility, movement competency, and basketball skills of one male collegiate basketball team $(\mathrm{n}=14$; mean age $=20.4$ \pm 1.6 years; level of competition ([“major American university"] not provided) and presented correlations with game variables based on these fitness measures. They reported positive correlations between lower extremity power (as measured by the standing long jump [SLJ] test) and minutes played, rebounds per game, and blocks per game. ${ }^{13}$ In addition, increasing torso stiffness, greater hip range of motion, and bench press performance also correlated with better performance measures. ${ }^{13}$ One characteristic, grip strength in the left hand, correlated negatively with performance measures. ${ }^{13}$ Ben Abdelkrim et al. ${ }^{1}$ assessed measures of lower extremity power, anthropometric measures, speed, and agility in male Tunisian national basketball team members ( $\mathrm{n}=$ 45; 3 teams assessed: U-18, U-20, and Senior levels). Point guards were significantly faster in short distance runs and had better agility. Power forwards and centers had shorter vertical jump heights than players in other positions; how- 
ever, they were significantly stronger than other positional players during bench press testing. ${ }^{1}$ Ostojic et al. ${ }^{7}$ assessed measures of aerobic fitness, anthropometrics, and power in five professional Serbian men's basketball teams $(n=60$; mean age $=23.4 \pm 3.5$ years). They reported that centers were significantly taller, had a greater percentage of body fat, and had lower maximal oxygen uptake, whereas guards were significantly older with a maximum lower heart rate. ${ }^{7}$ Due to the limited number of studies describing preseason testing protocols and relationships to performance measures or player position in men's collegiate basketball, additional studies are warranted.

Two FPTs that warrant assessment in the male collegiate basketball population are the SLJ and the single-leg hop (SLH) for distance test. The SLJ and SLH are FPTs that can serve as clinical correlates for measuring lower extremity strength and power. ${ }^{8,9,12,21-27}$ Both of these tests have historically been used to guide return to play decision making after an athletic injury to the lower quadrant (low back and/ or lower extremities). ${ }^{8,12,21,25,27}$ However, the SLJ and SLH have also been used to assess athletic readiness and to identify correlations between these measures and performance indicators. The SLJ has been used to measure strength and power in youth soccer players, ${ }^{28-30}$ collegiate baseball players, ${ }^{31}$ amateur orienteering athletes, ${ }^{32}$ collegiate basketball players, ${ }^{13}$ competitive hockey players, ${ }^{33-35}$ and National Football League football players. ${ }^{36}$ The SLH test has not been evaluated in athletes to the same extent as the SLJ test. The SLH test has been administered to identify muscular imbalance between lower extremities in non-injured populations. ${ }^{37}$

The purpose of this study was two-fold. The first was to report demographic information for National Association of Intercollegiate Athletics (NAIA) and National Collegiate Athletic Association (NCAA) Division III male collegiate basketball players. It was hypothesized that there would be no differences in demographic information, offseason training reports, and FPT measures between players based on level of competition. The second was to describe relationships between anthropometric measures and FPT measures. It was hypothesized that there would be no difference in relationships between anthropometric measures and preseason FPT performance between groups.

\section{METHODS}

\section{Participants}

Eighty-six male collegiate basketball players from six teams (NAIA, $\mathrm{n}=43$; Division III, $\mathrm{n}=43$ ) were recruited for this study. Participants were excluded from study par- ticipation if they were either younger than 18 years or restricted from sport participation at the time of testing by their primary provider. This study was approved by the Institutional Review Board of George Fox University. Participants were informed of the benefits and risks of the study prior to signing the institutionally approved informed consent document to participate in the study.

\section{Procedures}

The testing protocol consisted of the following: (1) athletes completed a demographic questionnaire, (2) height (cloth tape fixed to wall) and weight (standard medical scale) measures were collected, (3) each athlete performed a 5-minute dynamic warm-up, and (4) each participant performed of a total of nine maximal effort jumps: 3 SLJ and 3 SLH per lower extremity.

\section{Study Questionnaire}

Each athlete completed a questionnaire, prior to FPT testing, collecting the following information: age, years in college/university, age starting sport, and weekly time devoted to training during the 6-week period of time prior to the start of the official preseason. Athletes were asked to record the amount of time per week devoted to weightlifting, cardiovascular exercise (eg, distance running or cycling), plyometric exercise, and scrimmaging.

\section{Dynamic Warm-up}

Each athlete performed a dynamic warm-up prior to jump and hop testing. The purpose of having athletes perform a dynamic warm-up was to metabolically prepare the musculoskeletal and nervous systems for the physical rigor associated with maximal performance testing. ${ }^{38}$ This 5-minute warm-up consisted of performing active lower extremity movements across the width of a basketball court two to three times per each of the following: forward lunge walking, backward lunge walking, high knee marching, heel walking, and tiptoe walking. ${ }^{14,15}$ Three submaximal SLJs were also performed as part of the warm-up.

\section{SLJ}

Each athlete performed three maximal effort SLJs. Athletes stood with feet positioned shoulder width apart behind the starting line (a piece of tape). Athletes were instructed to clasp their hands behind their back, to jump (for distance) as far as possible, and to stick the landing for 5 seconds. ${ }^{21}$ Clasping the hands behind the back was performed to reduce the potential contribution of an arm 


\begin{tabular}{|c|c|c|c|c|}
\hline \multicolumn{5}{|c|}{$\begin{array}{c}\text { TABLE } 1 \\
\begin{array}{c}\text { Demographic Characteristics and Normalized Functional Performance Test } \\
\text { Measures (Mean } \pm \text { SD) for Male Collegiate Basketball Players }\end{array}\end{array}$} \\
\hline Characteristic & Total $(\mathrm{N}=\mathbf{8 6})$ & NAIA $(n=43)$ & NCAA Division III $(n=43)$ & $p a, b$ \\
\hline Age $(y)$ & $20.0 \pm 1.8$ & $20.7 \pm 2.0$ & $19.3 \pm 1.4$ & $\leq .0001$ \\
\hline Years in school & $2.2 \pm 1.2$ & $2.7 \pm 1.3$ & $1.8 \pm 0.9$ & .001 \\
\hline Age starting sport (y) & $8.6 \pm 2.9$ & $8.7 \pm 3.2$ & $8.4 \pm 2.6$ & .30 \\
\hline \multicolumn{5}{|l|}{ Off-season training (hr/wk) } \\
\hline Weightlifting & $4.7 \pm 2.3$ & $4.3 \pm 2.5$ & $5.2 \pm 2.0$ & .05 \\
\hline Cardiovascular exercise & $5.5 \pm 4.1$ & $7.0 \pm 4.4$ & $4.0 \pm 3.1$ & $\leq .0001$ \\
\hline Plyometric exercise & $2.2 \pm 2.0$ & $2.5 \pm 2.2$ & $1.9 \pm 1.8$ & .10 \\
\hline Scrimmage & $5.5 \pm 3.0$ & $5.7 \pm 3.6$ & $5.3 \pm 2.1$ & .60 \\
\hline Height $(m)$ & $1.88 \pm 0.07$ & $1.87 \pm 0.08$ & $1.89 \pm 0.07$ & .30 \\
\hline Weight (kg) & $83.6 \pm 9.5$ & $81.9 \pm 9.2$ & $85.4 \pm 9.6$ & .08 \\
\hline $\mathrm{BMI}\left(\mathrm{kg} / \mathrm{m}^{2}\right)$ & $23.7 \pm 2.0$ & $23.4 \pm 2.1$ & $24.0 \pm 2.0$ & .20 \\
\hline \multicolumn{5}{|c|}{ Functional performance tests (normalized to height) } \\
\hline Standing long jump & $1.0 \pm 0.1$ & $1.0 \pm 0.1$ & $1.0 \pm 0.1$ & .50 \\
\hline Right single-leg hop & $0.84 \pm 0.1$ & $0.86 \pm 0.1$ & $0.82 \pm 0.1$ & .07 \\
\hline Left single-leg hop & $0.85 \pm 0.1$ & $0.89 \pm 0.1$ & $0.81 \pm 0.1$ & .008 \\
\hline $\begin{array}{l}\mathrm{SD}=\text { standard deviation; NAIA = } \\
\text { alndependent } t \text { test. } \\
\text { bComparison between NAIA and } \\
\text { Values in bold are statistically sig }\end{array}$ & tics; $\mathrm{NCAA}=1$ & giate Athletic & ion; BMI = body mass index & \\
\hline
\end{tabular}

swing to one's overall distance. Ashby and Heegaard ${ }^{39}$ found an arm swing increased SLJ distances by $21 \%$. A trial was repeated if the athlete was unable to stick the landing and/or if the participant swung his arms during the test. The distance jumped was measured from the starting line to the rearmost heel. The mean of three SLJ scores, normalized to one's height, was used for data analyses.

\section{SLH}

Each athlete performed three maximal effort SLHs for each lower extremity. A coin-toss determined which leg was hopped off first with each successive trial alternating between lower extremities. Athletes were instructed to clasp their hands behind their back, to hop (for distance) as far as possible, and to stick the landing for 5 seconds. ${ }^{21}$ A trial was repeated if the athlete failed to stick the landing or if he swung his arms. The mean of three SLH scores, normalized to one's height, was used for data analyses.

\section{Statistical Analyses}

Means ( \pm standard deviation) were calculated for demographic characteristics, off-season training reports, anthropometric measures, and FPT measures. Mean FPT measures were normalized as a percentage of body height.
Independent $t$ tests were calculated to compare demographic characteristics, training habits, anthropometric measures, and FPT measures based on level of competition (NAIA vs Division III) or by starter status or player position. Anthropometric measures were categorized as ( -1 standard deviation [shortest, lightest, lowest] / mean [average] / +1 standard deviation [tallest, heaviest, or highest]). Analysis of variance (ANOVA) was performed to assess mean differences within groups for age and anthropometric measures. An a priori test-retest reliability for the FPTs was performed using intraclass correlation coefficients and has been reported previously (SLJ $=0.96[95 \%$ confidence interval $[\mathrm{CI}]$ : 0.83 to 0.97 ]; right $\mathrm{SLH}=0.95$ [95\% CI: 0.89 to 0.98 ]; left SLH $=0.96$ [95\% CI: 0.89 to 0.98$]$ ]. ${ }^{15}$ Data analysis was performed using SPSS statistical software (version 23; SPSS, Inc., Chicago, IL) with an alpha level set at 0.05.

\section{RESULTS}

Table 1 presents demographic characteristics, off-season training reports, and normalized FPT measures. The mean age for all participants was $20 \pm 1.8$ years (range: 18 to 25 years). The mean height for all participants was $1.88 \pm 0.07$ $\mathrm{m}$, the mean weight was $83.6 \pm 9.5 \mathrm{~kg}$, and the mean body mass index (BMI) was $23.7 \pm 2.0 \mathrm{~kg} / \mathrm{m}^{2}$. NAIA basket- 


\begin{tabular}{|c|c|c|c|c|c|c|c|c|c|}
\hline \multicolumn{10}{|c|}{$\begin{array}{l}\text { TA B LE } 2 \\
\text { Normalized Standing Long Jump and Single-Leg Hop (Mean } \pm \text { SD) Distances by } \\
\text { Age and Anthropometric Measures for All Male Collegiate Basketball Players }\end{array}$} \\
\hline \multirow[b]{2}{*}{ Variable } & \multicolumn{3}{|c|}{ Standing Long Jump } & \multicolumn{3}{|c|}{ Right Single-Leg Hop } & \multicolumn{3}{|c|}{ Left Single-Leg Hop } \\
\hline & $\mathbf{N}$ & Mean \pm SD & $P^{a}$ & $\mathbf{N}$ & Mean \pm SD & $P^{a}$ & $\mathbf{N}$ & Mean \pm SD & $P^{a}$ \\
\hline \multicolumn{10}{|l|}{ Age (y) } \\
\hline 18 & 21 & $0.99 \pm 0.10$ & .50 & 21 & $0.79 \pm 0.14$ & .10 & 21 & $0.81 \pm 0.15$ & .20 \\
\hline 19 & 22 & $1.02 \pm 0.10$ & & 22 & $0.87 \pm 0.11$ & & 22 & $0.87 \pm 0.13$ & \\
\hline 20 & 13 & $0.98 \pm 0.12$ & & 13 & $0.82 \pm 0.11$ & & 13 & $0.83 \pm 0.14$ & \\
\hline 21 and older & 30 & $0.98 \pm 0.11$ & & 30 & $0.86 \pm 0.12$ & & 30 & $0.88 \pm 0.11$ & \\
\hline \multicolumn{10}{|l|}{ Height (m) } \\
\hline Shortest (-1 SD) & 18 & $1.05 \pm 0.09^{b}$ & $\leq .0001$ & 18 & $0.87 \pm 0.14^{d}$ & .02 & 18 & $0.84 \pm 0.12$ & .20 \\
\hline Average & 50 & $1.00 \pm 0.09^{c}$ & & 50 & $0.85 \pm 0.11^{\mathrm{e}}$ & & 50 & $0.87 \pm 0.14$ & \\
\hline Tallest (+1 SD) & 18 & $0.90 \pm 0.17^{b, c}$ & & 18 & $0.77 \pm 0.13^{\mathrm{d}, \mathrm{e}}$ & & 18 & $0.80 \pm 0.13$ & \\
\hline \multicolumn{10}{|l|}{ Weight (kg) } \\
\hline Lightest (-1 SD) & 14 & $1.05 \pm 0.09^{f}$ & $\leq .0001$ & 14 & $0.91 \pm 0.09^{f}$ & $\leq .0001$ & 14 & $0.91 \pm 0.09^{f}$ & $\leq .0001$ \\
\hline Average & 59 & $1.00 \pm 0.099$ & & 59 & $0.85 \pm 0.11^{h}$ & & 59 & $0.86 \pm 0.12^{i}$ & \\
\hline Heaviest (+1 SD) & 13 & $0.88 \pm 0.12^{\mathrm{fg}}$ & & 13 & $0.72 \pm 0.13^{f, h}$ & & 13 & $0.72 \pm 0.15$ & \\
\hline \multicolumn{10}{|l|}{ BMI } \\
\hline Lowest (-1 SD) & 11 & $1.00 \pm 0.10$ & .02 & 11 & $0.87 \pm 0.09^{k}$ & .003 & 11 & $0.90 \pm 0.09^{\prime}$ & $\leq .0001$ \\
\hline Average & 61 & $1.00 \pm 0.09^{c}$ & & 61 & $0.86 \pm 0.11^{\mathrm{m}}$ & & 61 & $0.87 \pm 0.12^{j}$ & \\
\hline Highest (+1 SD) & 14 & $0.92 \pm 0.13^{c}$ & & 14 & $0.74 \pm 0.14^{\mathrm{k}, \mathrm{m}}$ & & 14 & $0.73 \pm 0.15^{\mathrm{j}, \mathrm{l}}$ & \\
\hline $\begin{array}{l}\text { SD = standard deviation } \\
\text { aAnalysis of variance. } \\
\text { 'Difference between sh } \\
\text { DDifference between av } \\
\text { dDifference between sh } \\
\text { 'Difference between av } \\
\text { 'Difference between ligh } \\
\text { 'D Difference between av } \\
\text { "Difference between av } \\
\text { 'Difference between ave } \\
\text { 'Difference between ave } \\
\text { 'Difference between lov } \\
\text { 'Difference between low } \\
\text { mDifference between av } \\
\text { Values in bold are statist }\end{array}$ & $\begin{array}{l}\text { body } \\
1 \text { SD) a } \\
\text { id talle } \\
1 \text { SD) a } \\
\text { id talle } \\
\text { SD) an } \\
\text { hd hea } \\
\text { hd hea } \\
\text { d heav } \\
\text { d high } \\
\text { SD) an } \\
\text { SD) an } \\
\text { nd hig } \\
\text { gnifica }\end{array}$ & $\begin{array}{l}\text { ndex } \\
\text { Illest (+1 SD) heigh } \\
\text { SD) heights; } P=\text {. } \\
\text { llest (+1 SD) heigh } \\
\text { I SD) heights; } P=\text {. } \\
\text { aviest (+1 SD) weig } \\
(+1 \text { SD) weight gro } \\
(+1 \text { SD) weight gro } \\
+1 \text { SD) weight gro } \\
-1 \text { SD) BMI groups; } \\
\text { nest (+1 SD) BMI g } \\
\text { est (+1 SD) BMI gr } \\
+1 \text { SD) BMI groups }\end{array}$ & $\begin{array}{l}P \leq .0001 \text { pos } \\
\text { post-hoc. } \\
P=.04 \text { post- } \\
\text { post-hoc. } \\
\text { groups } P \leq . \\
\text { s; } P \leq .0001 \mathrm{p} \\
\mathrm{s} ; P=.002 \mathrm{po} \\
\mathrm{s} ; P=.001 \mathrm{po} \\
=.02 \text { post-ho } \\
\text { pss; } P=.02 \mathrm{pc} \\
\text { ps; } P=.002 . \\
=.003 \text { post-h }\end{array}$ & $\begin{array}{l}\text { oc. } \\
\text { oc. } \\
1 \text { post } \\
\text { hoc. } \\
\text { oc. } \\
\text { oc. } \\
\text { hoc. }\end{array}$ & & & & & \\
\hline
\end{tabular}

ball players were significantly older $(P \leq .0001)$, had been enrolled in college longer $(P=.001)$, reported more time devoted to weekly cardiovascular exercises $(P \leq .0001)$, and had a farther left SLH $(P=.008)$ than their Division III counterparts. Division III basketball players reported more time devoted to off-season weightlifting $(P=.05)$ than their NAIA counterparts.

Tables 2-4 present relationships between demographic characteristics and normalized SLJ and SLH measures. Table 2 presents data for all $(n=86)$ male collegiate basketball players. In general, basketball players who were in the shorter or average height category, who were in the lighter or average weight category, or who were in the lowest or average BMI category jumped and/or hopped significantly farther than athletes in the taller, heavier, or highest BMI categories. Similar relationships were observed when analyzing participants per level of competition (Tables 3-4).

Table 5 presents comparisons of demographics, offseason training reports, and FPT measures by starter status both within group (eg, per level of competition) and between groups (eg, comparisons between NAIA and Division III players). There were few within-group differences between starters and non-starters at either level. Starters at the NAIA level were significantly older $(P=.03)$ than NAIA non-starters. The BMI of Division III non-starters was significantly greater than Division III starters $(P=.05)$. 
TABLE 3

Normalized Standing Long Jump and Single-Leg Hop (Mean \pm SD) Distances by Age and Anthropometric Measures for Male NAIA Basketball Players

\begin{tabular}{|c|c|c|c|c|c|c|c|c|c|}
\hline \multirow[b]{2}{*}{ Variable } & \multicolumn{3}{|c|}{ Standing Long Jump } & \multicolumn{3}{|c|}{ Right Single-Leg Hop } & \multicolumn{3}{|c|}{ Left Single-Leg Hop } \\
\hline & $\mathbf{N}$ & Mean \pm SD & $P^{a}$ & $\mathbf{N}$ & Mean \pm SD & $p^{a}$ & $\mathbf{N}$ & Mean \pm SD & $p^{a}$ \\
\hline \multicolumn{10}{|l|}{ Age (y) } \\
\hline 18 & 6 & $1.03 \pm 0.11$ & \multirow{4}{*}{.90} & 6 & $0.84 \pm 0.11$ & & 6 & $0.85 \pm 0.08$ & \\
\hline 19 & 10 & $1.02 \pm 0.09$ & & 10 & $0.89 \pm 0.08$ & \multirow{3}{*}{.05} & 10 & $0.91 \pm 0.09$ & \multirow{3}{*}{.30} \\
\hline 20 & 5 & $1.02 \pm 0.15$ & & 5 & $0.88 \pm 0.05$ & & 5 & $0.95 \pm 0.07$ & \\
\hline 21 and older & 22 & $0.97 \pm 0.10$ & & 22 & $0.85 \pm 0.13$ & & 22 & $0.87 \pm 0.11$ & \\
\hline \multicolumn{10}{|l|}{ Height (m) } \\
\hline Shortest (-1 SD) & 10 & $1.07 \pm 0.10^{b}$ & \multirow{3}{*}{.005} & 10 & $0.92 \pm 0.08^{c}$ & \multirow{3}{*}{.005} & 10 & $0.90 \pm 0.06$ & \multirow{3}{*}{.06} \\
\hline Average & 24 & $1.00 \pm 0.10$ & & 24 & $0.87 \pm 0.09$ & & 24 & $0.91 \pm 0.10$ & \\
\hline Tallest (+1 SD) & 9 & $0.92 \pm 0.10^{b}$ & & 9 & $0.77 \pm 0.14^{c}$ & & 9 & $0.81 \pm 0.14$ & \\
\hline \multicolumn{10}{|l|}{ Weight (kg) } \\
\hline Lightest (-1 SD) & 9 & $1.07 \pm 0.08^{d}$ & \multirow{3}{*}{.002} & 9 & $0.91 \pm 0.10^{f}$ & \multirow{3}{*}{.02} & 9 & $0.93 \pm 0.09^{9}$ & \multirow{3}{*}{.04} \\
\hline Average & 26 & $1.00 \pm 0.08^{\mathrm{e}}$ & & 26 & $0.87 \pm 0.09$ & & 26 & $0.90 \pm 0.07$ & \\
\hline Heaviest (+1 SD) & 8 & $0.90 \pm 0.12^{\mathrm{d}, \mathrm{e}}$ & & 8 & $0.78 \pm 0.13^{f}$ & & 8 & $0.81 \pm 0.15^{\mathrm{g}}$ & \\
\hline \multicolumn{10}{|l|}{ BMI } \\
\hline Lowest (-1 SD) & 5 & $0.98 \pm 0.07$ & \multirow{3}{*}{.05} & 5 & $0.86 \pm 0.11$ & \multirow{3}{*}{.10} & 5 & $0.91 \pm 0.12^{h}$ & \multirow{3}{*}{.04} \\
\hline Average & 31 & $1.02 \pm 0.09$ & & 31 & $0.88 \pm 0.09$ & & 31 & $0.90 \pm 0.08$ & \\
\hline Highest (+1 SD) & 7 & $0.92 \pm 0.14$ & & 7 & $0.79 \pm 0.15$ & & 7 & $0.80 \pm 0.15^{\mathrm{h}}$ & \\
\hline \multicolumn{10}{|c|}{ 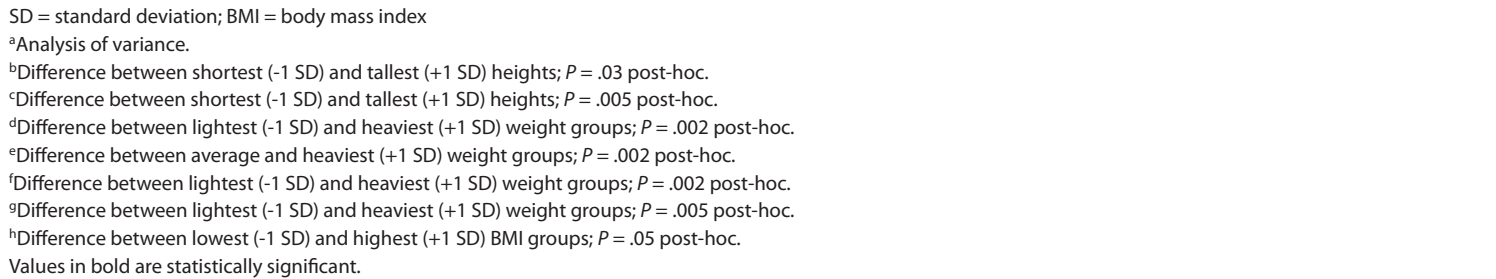 } \\
\hline
\end{tabular}

Division III starters hopped with the left lower extremity significantly farther than non-starters $(P=.01)$. There were also few significant differences (age and years in college) between starters based on level of competition. In other words, NAIA and Division III starters reported similar off-season training reports and presented with similar FPT scores. The greatest number of significant differences was observed when comparing non-starters based on level of competition. NAIA non-starters were older $(P=.03)$, devoted more time to cardiovascular exercise in the off-season $(P=.002)$, were lighter $(P=.04)$, and hopped significantly farther (right lower extremity, $P=.01$; left lower extremity, $P=.001)$ than their Division III counterparts.

Table 6 presents comparison of demographics, offseason training reports, and FPT measures by player position (eg, guards vs forwards/centers) and per level of competition. NAIA forwards and centers reported more time devoted to plyometric exercise during the off-season $(P=.03)$ than their guard counterparts; however, this did not relate to significantly greater jump or hop distances. Rather, guards jumped significantly farther than forwards/centers $(P=.02)$. Similar FPT findings were observed within the Division III population. Division III guards jumped (SLJ) and hopped (SLH) significantly farther than the forwards/centers (SLJ, $P$ $=.004$; right SLH, $P=.05$, left SLH, $P=.04$ ). When comparing between level of competition by position, NAIA guards were older and spent more time performing cardiovascular exercise in the off-season, whereas Division III guards devoted more off-season training time to weightlifting; however, there were no differences in FPT measures. Similar relationships were observed 
TABLE 4

Normalized Standing Long Jump and Single-Leg Hop (Mean \pm SD) Distances by Age and Anthropometric Measures for Male NCAA Division III Basketball Players

\begin{tabular}{|c|c|c|c|c|c|c|c|c|c|}
\hline \multirow[b]{2}{*}{ Variable } & \multicolumn{3}{|c|}{ Standing Long Jump } & \multicolumn{3}{|c|}{ Right Single-Leg Hop } & \multicolumn{3}{|c|}{ Left Single-Leg Hop } \\
\hline & $\mathbf{N}$ & Mean \pm SD & $P^{a}$ & $\mathbf{N}$ & Mean \pm SD & $P^{a}$ & $\mathbf{N}$ & Mean \pm SD & $P^{a}$ \\
\hline \multicolumn{10}{|l|}{ Age (y) } \\
\hline 18 & 15 & $0.98 \pm 0.10$ & \multirow{4}{*}{.80} & 15 & $0.77 \pm 0.15$ & \multirow{4}{*}{.20} & 15 & $0.79 \pm 0.17$ & \multirow{4}{*}{.20} \\
\hline 19 & 12 & $1.01 \pm .011$ & & 12 & $0.85 \pm 0.13$ & & 12 & $0.83 \pm 0.15$ & \\
\hline 20 & 8 & $0.96 \pm 0.11$ & & 8 & $0.78 \pm 0.12$ & & 8 & $0.74 \pm 0.11$ & \\
\hline 21 and older & 8 & $0.98 \pm 0.13$ & & 8 & $0.89 \pm 0.12$ & & 8 & $0.90 \pm 0.11$ & \\
\hline \multicolumn{10}{|l|}{ Height (m) } \\
\hline Shortest (-1 SD) & 8 & $1.03 \pm 0.09$ & \multirow{3}{*}{.20} & 8 & $0.81 \pm 0.17$ & \multirow{3}{*}{.90} & 8 & $0.77 \pm 0.14$ & \multirow{3}{*}{.70} \\
\hline Average & 30 & $0.98 \pm 0.10$ & & 30 & $0.82 \pm 0.13$ & & 30 & $0.82 \pm 0.15$ & \\
\hline Tallest (+1 SD) & 5 & $0.91 \pm 0.15$ & & 5 & $0.80 \pm 0.14$ & & 5 & $0.82 \pm 0.14$ & \\
\hline \multicolumn{10}{|l|}{ Weight (kg) } \\
\hline Lightest (-1 SD) & 7 & $1.01 \pm 0.09$ & \multirow{3}{*}{.02} & 7 & $0.86 \pm 0.12$ & \multirow{3}{*}{.20} & 7 & $0.86 \pm 0.14$ & \multirow{3}{*}{.20} \\
\hline Average & 28 & $1.00 \pm 0.09^{b}$ & & 28 & $0.83 \pm 0.13$ & & 28 & $0.83 \pm 0.14$ & \\
\hline Heaviest (+1 SD) & 8 & $0.89 \pm 0.14^{b}$ & & 8 & $0.74 \pm 0.15$ & & 8 & $0.73 \pm 0.17$ & \\
\hline \multicolumn{10}{|l|}{ BMI } \\
\hline Lowest (-1 SD) & 7 & $0.95 \pm 0.08$ & \multirow{3}{*}{.03} & 7 & $0.82 \pm 0.17^{d}$ & \multirow{3}{*}{.006} & 7 & $0.84 \pm 0.19^{f}$ & \multirow{3}{*}{.003} \\
\hline Average & 31 & $1.01 \pm 0.10^{c}$ & & 31 & $0.84 \pm 0.11^{\mathrm{e}}$ & & 31 & $0.84 \pm 0.12^{\mathrm{g}}$ & \\
\hline Highest (+1 SD) & 5 & $0.88 \pm 0.11^{c}$ & & 5 & $0.64 \pm 0.13^{\mathrm{d}, \mathrm{e}}$ & & 5 & $0.61 \pm 0.11^{f, g}$ & \\
\hline \multicolumn{10}{|c|}{$\begin{array}{l}\mathrm{SD}=\text { standard deviation; } \mathrm{BMI}=\text { body mass index } \\
\text { aAnalysis of variance. } \\
\text { 'Difference between average and heaviest (+1 SD) weight groups; } P=.02 \text { post-hoc. } \\
\text { 'Difference between average and highest (+1 SD) BMI groups; } P=.03 \text { post-hoc. } \\
\text { 'Difference between lowest ( }-1 \mathrm{SD} \text { ) and highest (+1 SD) BMI groups; } P=.05 \text { post-hoc. } \\
\text { eDifference between average and highest (+1 SD) BMI groups; } P=.005 \text { post-hoc. } \\
\text { fDifference between lowest (-1 SD) and highest (+1 SD) BMI groups; } P=.01 \text { post-hoc. } \\
\text { 9Difference between average and highest (+1 SD) BMI groups; } P=.002 \text { post-hoc. } \\
\text { Values in bold are statistically significant. }\end{array}$} \\
\hline
\end{tabular}

when comparing forwards/centers. NAIA forwards/ centers were older and devoted more time to cardiovascular and plyometric exercise during the off-season. NAIA forwards/centers hopped [left SLH] significantly farther than Division III counterparts $(P=.02)$.

\section{DISCUSSION}

To the best of our knowledge, this is the first study to present SLJ and SLH data for male collegiate basketball players with specific comparisons based on off-season training habits, level of competition, position, and starter status. A unique feature of this study is that it presents data for NAIA and NCAA Division III athletes, populations that are underrepresented in the literature.

The data presented in this study can be used by coaches and athletic trainers to assess an athlete's readiness for sport. First, a coach or sports medicine professional can use these quick, easy-to-perform tests to compare the athlete's FPT measures to their counterparts at either the NAIA or Division III levels. For example, a strength coach or athletic trainer could evaluate in the month of May, prior to the end of the academic year, an athlete's SLJ and SLH measures. If those measures were deemed suboptimal, the strength coach could implement an individualized offseason (summer) training program for the athlete. Second, coaches may want to consider adjusting their training programs to improve jump and hop measures in forwards and centers. The taller/heavier athletes (eg, forwards and centers) had significantly shorter hop and jump measures. Because these FPTs are an indicator of lower extremity strength and power, it can be argued that the forwards and centers, who are responsible for rebounding and blocking shots, should be able to jump and hop equal if not greater distances than their shorter/lighter counterparts. To de- 


\begin{tabular}{|c|c|c|c|c|c|c|c|c|}
\hline \multicolumn{9}{|c|}{$\begin{array}{l}\text { Comparison of Demographics, Off-Season Training Habits, and } \\
\text { Functional Performance Test Measures (Mean } \pm \text { SD) for Starters and Non-starters } \\
\text { Within and Between Collegiate Levels of Basketball Competition }\end{array}$} \\
\hline Characteristic & $\begin{array}{c}\text { NAIA } \\
\text { Starters } \\
(n=15)\end{array}$ & $\begin{array}{c}\text { NAIA } \\
\text { Non-starters } \\
(\mathbf{n}=\mathbf{2 8})\end{array}$ & $p^{a}$ & $\begin{array}{l}\text { NCAA } \\
\text { Division } \\
\text { III } \\
\text { Starters } \\
(\mathbf{n}=15)\end{array}$ & $\begin{array}{l}\text { NCAA } \\
\text { Division III } \\
\text { Non-starters } \\
(\mathbf{n}=\mathbf{2 8})\end{array}$ & $P^{b}$ & $\begin{array}{l}\text { Difference } \\
\text { Between NAIA } \\
\text { and NCAA } \\
\text { Division III } \\
\text { Starters' }\end{array}$ & $\begin{array}{l}\text { Difference } \\
\text { Between } \\
\text { NAIA \& NCAA } \\
\text { Division III } \\
\text { Non-starters }\end{array}$ \\
\hline \multicolumn{9}{|l|}{ Non-starters ${ }^{d}$} \\
\hline Age (y) & $21.6 \pm 1.5$ & $20.2 \pm 2.1$ & .03 & $19.6 \pm 1.7$ & $19.2 \pm 1.2$ & .40 & .002 & .03 \\
\hline Year in school & $3.4 \pm 1.1$ & $2.3 \pm 1.2$ & .004 & $2.0 \pm 1.1$ & $1.8 \pm 0.9$ & .40 & .002 & .08 \\
\hline Age starting sport (y) & $7.8 \pm 3.1$ & $9.2 \pm 3.3$ & .20 & $7.5 \pm 3.0$ & $8.9 \pm 2.3$ & .10 & .80 & .70 \\
\hline \multicolumn{9}{|l|}{ Off-season training (hr/wk) } \\
\hline Weightlifting & $4.2 \pm 2.2$ & $4.3 \pm 2.7$ & .90 & $5.1 \pm 1.9$ & $5.3 \pm 2.0$ & .80 & .20 & .10 \\
\hline Cardiovascular exercise & $6.7 \pm 3.8$ & $7.2 \pm 4.7$ & .70 & $4.6 \pm 2.9$ & $3.7 \pm 3.2$ & .40 & .10 & .002 \\
\hline Plyometric exercise & $2.7 \pm 2.5$ & $2.4 \pm 2.0$ & .60 & $2.5 \pm 2.6$ & $1.6 \pm 1.1$ & .10 & .80 & .07 \\
\hline Scrimmage & $5.5 \pm 3.6$ & $5.8 \pm 3.8$ & .80 & $5.3 \pm 2.1$ & $5.3 \pm 2.2$ & .90 & .90 & .50 \\
\hline Height $(\mathrm{m})$ & $1.89 \pm 0.08$ & $1.86 \pm 0.07$ & .30 & $1.89 \pm 0.07$ & $1.88 \pm 0.07$ & .60 & .80 & .20 \\
\hline Weight (kg) & $82.9 \pm 9.1$ & $81.3 \pm 9.4$ & .60 & $83.2 \pm 8.9$ & $86.6 \pm 9.8$ & .30 & .90 & .04 \\
\hline $\operatorname{BMI}\left(\mathrm{kg} / \mathrm{m}^{2}\right)$ & $23.3 \pm 2.2$ & $23.5 \pm 2.1$ & .80 & $23.2 \pm 1.9$ & $24.4 \pm 2.0$ & .05 & .80 & .10 \\
\hline \multicolumn{9}{|l|}{ FPTs } \\
\hline Standing long jump & $0.98 \pm 0.11$ & $1.00 \pm 0.10$ & .50 & $1.00 \pm 0.10$ & $0.97 \pm 0.11$ & .30 & .50 & .20 \\
\hline Right single-leg hop & $0.85 \pm 0.09$ & $0.87 \pm 0.11$ & .50 & $0.87 \pm 0.14$ & $0.79 \pm 0.13$ & .06 & .70 & .01 \\
\hline Left single-leg hop & $0.90 \pm 0.08$ & $0.88 \pm 0.11$ & .70 & $0.89 \pm 0.15$ & $0.77 \pm 0.14$ & .01 & .90 & .001 \\
\hline \multicolumn{9}{|c|}{ 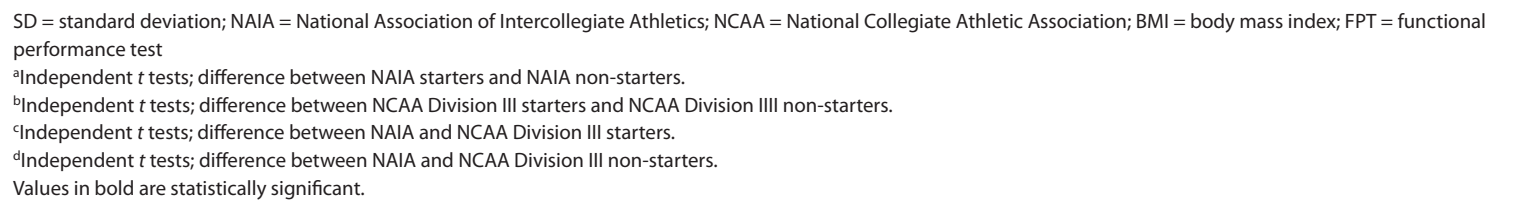 } \\
\hline
\end{tabular}

termine optimal jump and hop profiles for forwards and centers future studies should correlate preseason FPT measures with rebounding and blocks statistics.

Comparisons of demographic measures, training reports, and FPT scores between athletes based on levels of competition is also a unique feature to this study that can potentially be of value for coaches and athletic trainers who work with current or future collegiate athletes. For example, a Division III coach may want to compare his or her team's off-season training habits against normative data for Division III or NAIA athletes. It is interesting that NAIA athletes reported performing $7.0 \pm 4.4 \mathrm{hr} / \mathrm{wk}$ of cardiovascular exercise (eg, aerobic forms of exercise such as running or cycling), whereas Division III players only reported 4.0 $\pm 3.1 \mathrm{hr} / \mathrm{wk}$. Increasing cardiovascular training requirements may have benefits for endurance capacity and altering weight and/or BMI (eg, Division III non-starters were significantly heavier than their NAIA counterparts and Division III non-starters had a significantly greater BMI than Division III starters). Strength training professionals who work with high school basketball players can use these data to compare their athlete's performance with normative data from NAIA and Division III levels.

This data set can also be used by athletic trainers and other sports medicine professionals when assessing an athlete's readiness to return to sport after an injury. The SLJ and SLH tests are frequently used in rehabilitation to assess the effectiveness of a rehabilitation program and to help guide discharge back to sport. ${ }^{12,25,27}$ Davies and Zillmer ${ }^{21}$ suggested that male athletes should be able to jump for distances at least $90 \%$ of their height and should hop for distances at least $80 \%$ of their height prior to returning to sport. Based on the data from this study, it may be warranted to require male collegiate basketball players to jump 


\section{Comparison of FPT Measures (Mean \pm SD) for Guards and Forwards Within and Between Collegiate Levels of Basketball Competition}

\begin{tabular}{|c|c|c|c|c|c|c|c|c|}
\hline Characteristic & $\begin{array}{c}\text { NAIA } \\
\text { Guards } \\
(n=27)\end{array}$ & $\begin{array}{c}\text { NAIA } \\
\text { Forwards } \\
\text { \& Centers } \\
(n=16)\end{array}$ & $P^{a}$ & $\begin{array}{c}\text { NCAA } \\
\text { Division III } \\
\text { Guards } \\
(n=26)\end{array}$ & $\begin{array}{c}\text { NCAA } \\
\text { Division III } \\
\text { Forwards \& } \\
\text { Centers } \\
(n=17)\end{array}$ & $P^{b}$ & $\begin{array}{l}\text { Difference } \\
\text { Between } \\
\text { NAIA and } \\
\text { NCAA } \\
\text { Division III } \\
\text { Guardsc }\end{array}$ & $\begin{array}{c}\text { Difference } \\
\text { Between } \\
\text { NAIA and } \\
\text { NCAA } \\
\text { Division III } \\
\text { Forwards \& } \\
\text { Centers }^{d}\end{array}$ \\
\hline Age (y) & $20.5 \pm 1.9$ & $21.0 \pm 2.1$ & .05 & $19.5 \pm 1.5$ & $19.0 \pm 1.1$ & .20 & .05 & .002 \\
\hline Year in school & $2.6 \pm 1.3$ & $2.8 \pm 1.3$ & .70 & $2.0 \pm 1.0$ & $1.6 \pm 0.8$ & .20 & .07 & .005 \\
\hline Age starting sport (y) & $8.7 \pm 3.2$ & $8.7 \pm 3.4$ & .90 & $8.4 \pm 2.7$ & $8.4 \pm 2.7$ & .90 & .70 & .80 \\
\hline \multicolumn{9}{|c|}{ Off-season training ( $\mathrm{hr} / \mathrm{wk})$} \\
\hline Weightlifting & $4.1 \pm 2.6$ & $4.4 \pm 2.4$ & .70 & $5.6 \pm 1.9$ & $4.6 \pm 1.9$ & .10 & .03 & .80 \\
\hline $\begin{array}{l}\text { Cardiovascular } \\
\text { exercise }\end{array}$ & $7.3 \pm 4.8$ & $6.5 \pm 3.7$ & .60 & $4.1 \pm 3.1$ & $3.9 \pm 3.2$ & .90 & .006 & .04 \\
\hline Plyometric exercise & $2.0 \pm 2.0$ & $3.4 \pm 2.2$ & .03 & $1.9 \pm 1.3$ & $1.8 \pm 2.4$ & .90 & .90 & .05 \\
\hline Scrimmage & $6.1 \pm 3.8$ & $4.9 \pm 3.4$ & .30 & $4.9 \pm 2.1$ & $6.0 \pm 2.1$ & .10 & .20 & .30 \\
\hline Height (m) & $1.83 \pm 0.06$ & $1.94 \pm 0.05$ & $\leq .0001$ & $1.85 \pm 0.06$ & $1.94 \pm 0.05$ & $\leq .0001$ & .20 & .70 \\
\hline Weight (kg) & $78.1 \pm 7.5$ & $88.2 \pm 8.5$ & $\leq .0001$ & $80.0 \pm 5.7$ & $93.7 \pm 8.4$ & $\leq .0001$ & .30 & .07 \\
\hline $\mathrm{BMI}\left(\mathrm{kg} / \mathrm{m}^{2}\right)$ & $23.3 \pm 1.8$ & $23.6 \pm 2.5$ & .70 & $23.4 \pm 1.9$ & $24.8 \pm 1.9$ & .02 & .90 & .10 \\
\hline \multicolumn{9}{|l|}{ FPTs } \\
\hline Standing long jump & $1.03 \pm 0.09$ & $0.95 \pm 0.11$ & .02 & $1.02 \pm 0.08$ & $0.93 \pm 0.12$ & .004 & .70 & .60 \\
\hline Right single-leg hop & $0.89 \pm 0.09$ & $0.83 \pm 0.13$ & .10 & $0.85 \pm 0.12$ & $0.77 \pm 0.15$ & .05 & .20 & .20 \\
\hline Left single-leg hop & $0.89 \pm 0.09$ & $0.88 \pm 0.13$ & .80 & $0.85 \pm 0.13$ & $0.76 \pm 0.16$ & .04 & .20 & .02 \\
\hline $\begin{array}{l}\mathrm{SD}=\text { standard deviation; NA } \\
\text { performance test } \\
\text { aIndependent } t \text { tests; differer } \\
\text { bIndependent } t \text { tests; differer } \\
\text { Independent } t \text { tests; differer } \\
\text { dIndependent } t \text { tests; differer } \\
\text { Values in bold are statisticalll }\end{array}$ & $\begin{array}{l}\text { National Associat } \\
\text { etween NAIA gua } \\
\text { etween Division } \\
\text { etween NAIA anc } \\
\text { etween NAIA anc } \\
\text { ificant. }\end{array}$ & $\begin{array}{l}\text { n of Intercollegiat } \\
\text { ds and NAIA forw } \\
\text { guards and NCA } \\
\text { NCAA Division III } \\
\text { NCAA Division III }\end{array}$ & $\begin{array}{l}\text { Athletics; NC } \\
\text { rds and cente } \\
\text { Division IIII fo } \\
\text { ards. } \\
\text { rwards and C }\end{array}$ & $\mathrm{A}=$ National Colle & giate Athletic Assoc & ation; BMI = & ody mass index; FF & $=$ functional \\
\hline
\end{tabular}

greater than $90 \%$ of their height and hop greater than $80 \%$ of their height prior to returning to sport after an injury.

Some limitations for this study have been mentioned previously. First, there is the potential for recall bias by the athletes when reporting their off-season training volumes. However, this study was conducted by a research team that was independent of any coaching staff. Thus, there would not be any reason for an athlete to purposefully over-inflate his off-season training reports. Second, relationships between preseason FPT measures and offseason training reports are presented; however, in the cases where there are significant differences in jump or hop distances based on training volumes, we are unable to suggest a causal relationship. A study using a pretestposttest design would be needed.

The SLJ and the SLH are two FPTs that can serve as a clinical correlate for lower extremity strength and power. Coaches and sports medicine professionals can use this normative data set to assess aspects of athletic readiness.

\section{IMPLICATIONS FOR CLINICAL PRACTICE}

Athletic trainers and coaches should use the SLJ and SLH tests to compare their athlete's performance against these normative values. A coach can individualize a training program to address deficits if an athlete's FPT measures are below mean scores. Athletic trainers and other sports medicine professionals can use these measures to help determine if an athlete is ready to return to sport after an injury.

\section{REFERENCES}

1. Ben Abdelkrim N, Chaouachi A, Chamari K, Chtara M, Castagna C. Positional role and competitive-level differences in elite-level men's basketball players. J Strength Cond Res. 2010;24:1346-1355. 
2. Bullock GS, Arnold TW, Plisky PJ, Butler RJ. Basketball players' dynamic performance across competition levels [published online ahead of print February 6, 2016]. J Strength Cond Res.

3. Castro-Piñero J, Ortega FB, Artero EG, et al. Assessing muscular strength in youth: usefulness of standing long jump as a general index of muscular fitness. J Strength Cond Res. 2010;24:1810-1817.

4. Chaouachi A, Brughelli M, Chamari K, et al. Lower limb maximal dynamic strength and agility determinants in elite basketball players. J Strength Cond Res. 2009;23:1570-1577.

5. Delextrat A, Cohen D. Physiological testing of basketball players: toward a standard evaluation of anaerobic fitness. J Strength Cond Res. 2008;22:1066-1072.

6. Metaxas TI, Koutlianos N, Sendelides T, Mandroukas A. Preseason physiological profile of soccer and basketball players in different divisions. J Strength Cond Res. 2009;23:1704-1713.

7. Ostojic SM, Mazic S, Dikic N. Profiling in basketball: physical and physiological characteristics of elite players. J Strength Cond Res. 2006;20:740-744.

8. Petschnig R, Baron R, Albrecht M. The relationship between isokinetic quadriceps strength test and hop tests for distance and onelegged vertical jump test following anterior cruciate ligament reconstruction. J Orthop Sports Phys Ther. 1998;28:23-31.

9. Pincivero DM, Lephart SM, Karunakara RG. Relation between open and closed kinematic chain assessment of knee strength and functional performance. Clin J Sport Med. 1997;7:11-16.

10. Puente C, Abián-Vicén J, Areces F, López R, Del Coso J. Physical and physiological demands of experienced male basketball players during a competitive game. J Strength Cond Res. 2017;31:956-962.

11. Barfield JP, Johnson RJ, Russo P, Cobler DC. Reliability and validity of the performance index evaluation among men's and women's college basketball players. J Strength Cond Res. 2007;21:643-645.

12. Manske RC, Prohaska D, Lucas B. Recent advances following anterior cruciate ligament reconstruction: rehabilitation perspectives: critical reviews in rehabilitation medicine. Curr Rev Musculoskelet Med. 2012;5:59-71.

13. McGill SM, Andersen JT, Horne AD. Predicting performance and injury resilience from movement quality and fitness scores in a basketball team over 2 years. J Strength Cond Res. 2012;26:1731-1739.

14. Brumitt J, Heiderscheit BC, Manske RC, Niemuth PE, Rauh MJ. Lower extremity functional tests and risk of injury in Division III collegiate athletes. Int J Sports Phys Ther. 2013;8:216-227.

15. Brumitt J, Heiderscheit BC, Manske RC, Niemuth PE, Rauh MJ. Off-season training habits and preseason functional test measures of Division III collegiate athletes: a descriptive report. Int J Sports Phys Ther. 2014;9:447-455.

16. Brumitt J, Heiderscheit BC, Manske RC, Niemuth P, Mattocks A, Rauh MJ. The lower extremity functional test and lower-quadrant injury in NCAA Division III athletes: a descriptive and epidemiologic report. J Sport Rehabil. 2016;25:219-226.

17. Brumitt J, Engilis A, Isaak D, Briggs A, Mattocks A. Preseason jump and hop measures in male collegiate basketball players: an epidemiologic report. Int J Sports Phys Ther. 2016;11:954-961.

18. Knapik JJ, Bauman CL, Jones BH, Harris J, Vaughan L. Preseason strength and flexibility imbalances associated with athletic injuries in female collegiate athletes. Am J Sports Med. 1991;19:76-81.

19. Payne KA, Berg K, Latin RW. Ankle injuries and ankle strength, flexibility, and proprioception in college basketball players. $J$ Athl Train. 1997;32:221-225.
20. Riva D, Bianchi R, Rocca F, Mamo C. Proprioceptive training and injury prevention in a professional men's basketball team: a six-year prospective study. J Strength Cond Res. 2016;30:461-475.

21. Davies GJ, Zillmer DA. Functional progression of a patient through a rehabilitation program. Orthop Phys Ther Clin NAm. 2000;9:103-117.

22. Guney H, Yuksel I, Kaya D, Doral MN. Correlation between quadriceps to hamstring ratio and functional outcomes in patellofemoral pain. Knee. 2016;23:610-615.

23. Lockie RG, Callaghan SJ, Berry SP, et al. Relationship between unilateral jumping ability and asymmetry on multidirectional speed in team-sport athletes. J Strength Cond Res. 2014;28:3557-3566.

24. Manske R, Reiman M. Functional performance testing for power and return to sports. Sports Health. 2013;5:244-250.

25. Manske RC, Davies GJ. Examination of the patellofemoral joint. Int J Sports Phys Ther. 2016;11:831-853.

26. Reiman MP, Manske RC. Functional Testing in Human Performance. Champaign, IL: Human Kinetics; 2009.

27. Wilk KE, Romaniello WT, Soscia SM, Arrigo CA, Andrews JR. The relationship between subjective knee scores, isokinetic testing, and functional testing in the ACL-reconstructed knee. J Orthop Sports Phys Ther. 1994;20:60-73.

28. Deprez D, Valente-Dos-Santos J, Coelho-E-Silva MJ, Lenoir M, Philippaerts R, Vaeyens R. Multilevel development models of explosive leg power in high-level soccer players. Med Sci Sports Exerc. 2015;47:1408-1415.

29. Hammami R, Chaouachi A, Makhlouf I, Granacher U, Behm DG. Associations between balance and muscle strength, power performance in male youth athletes of different maturity status. Pediatr Exerc Sci. 2016;28:521-534.

30. Re AH, Cattuzzo MT, Henrique Rdos S, Stodden DF. Physical characteristics that predict involvement with the ball in recreational youth soccer. J Sports Sci. 2016;34:1716-1722.

31. Lehman G, Drinkwater EJ, Behm DG. Correlation of throwing velocity to the results of lower-body field tests in male college baseball players. J Strength Cond Res. 2013;27:902-908.

32. Hébert-Losier K, Jensen K, Holmberg HC. Jumping and hopping in elite and amateur orienteering athletes and correlations to sprinting and running. Int J Sports Phys Perform. 2014;9:993-999.

33. Farlinger CM, Kruisselbrink D, Fowles JR. Relationships to skating performance in competitive hockey players. J Strength Cond Res. 2007;21:915-922

34. Runner AR, Lehnhard RA, Butterfield SA, Tu S, O'Neill T. Predictors of speed using off-ice measures of college hockey players. $J$ Strength Cond Res. 2016;30:1626-1632.

35. Vescovi JD, Murray TM, VanHeest JL. Positional performance profiling of elite ice hockey players. Int J Sports Physiol Perform. 2006;1:84-94.

36. Kuzmits FE, Adams AJ. The NFL combine: does it predict performance in the National Football League? J Strength Cond Res. 2008;22:1721-1727.

37. Jones PA, Bampouras TM. A comparison of isokinetic and functional methods of assessing bilateral strength imbalance. J Strength Cond Res. 2010;24:1553-1558.

38. Vetter RE. Effects of six warm-up protocols on sprint and jump performance. J Strength Cond Res. 2007;21:819-823.

39. Ashby BM, Heegaard JH. Role of arm motion in the standing long jump. J Biomech. 2002;35:1631-1637. 